\title{
Primary care on small islands: a unique challenge
}

For the purposes of this article 'small' is defined as a population that might reasonably be cared for by a single practitioner at any one time. Within the UK, such islands are situated principally to the West and North of the Scottish mainland (Hebrides, Orkney, Shetland) with populations ranging from about 60 to over 1000. Many of the practice issues are similar to those experienced by practices on larger islands, or indeed, 'remote and rural' practices in mainland UK. The uniqueness is defined by the need to access secondary care via an air or sea journey, and the need for cost-effective provision of a range of primary care services accessible to a small population.

Most islands with at least 200 people may have a GP and and district nurse. There may be some regular visiting services such as chiropody and health visiting.

The model of service provision has in many instances changed over recent years from GMS GPs to salaried GPs. Nurse practitioners are increasingly replacing GPs on islands with very small populations, and those whose sea/air journeys may be shorter and less vulnerable to changes in the weather. In Orkney, island GPs and nurse practitioners are increasingly working as part of a formal network to provide mutual support via email, telephone and videoconferencing.

Other innovations include remote and rural fellowships which allow GPs who have completed standard GP training to be funded for further supported practice in a remote and rural setting. The RCGP Remote and Rural Forum is in it's infancy and will undoubtedly contribute to supporting island practitioners. These initiatives could be strengthened by the development of GP registrar posts jointly between island and non island practices.

Mainland GPs with 'average' list sizes may be left wondering how a GP with a few hundred patients can possibly be kept busy. On many islands, the GP will provide care 24 hours a day 7 days a week for several weeks on end. The number of outof-hours calls will generally be few, but the need to be available at all times makes the jobs unattractive to many. However this continuity of care provides satisfaction for those who chose to work in island settings, and is often counterbalanced by reasonably generous remuneration and annual leave in return for this commitment.

Aside from the out-of-hours commitment, much of the work comes from taking on tasks which would normally be done by a practice nurse or others, and island GPs can be expected to take on some or all of the following tasks; minor injuries, prehospital emergency care, enhanced monitoring and follow-up of chronic disease under remote supervision from a specialist, phlebotomy, counselling, dispensing, palliative care, 'primary care' chronic disease monitoring - blood pressure, asthma, diabetes mellitus (that is, 'practice nurse roles') - and second ambulanceman. Some of these may be less attractive, while other roles reclaim territory which many GPs have been sorry to lose.

Support needed by island practitioners comes in many forms. Willing access to specialist advice via email, telephone, and videoconferencing is vital, particularly in emergencies and where evacuation of a casualty may take several hours. Local populations must continue to use out-ofhours services appropriately when the GP is on call for several days at a stretch, and take their 'public health' obligations seriously in regard to such issues as drink driving, the wearing of seat belts and the maintenance of motor vehicles in areas where there is no police to monitor compliance with the law.

Other barriers range from personal issues (for example, commitment from spouse and family to island life) to national policy which frequently tries to apply policy designed to tackle the problems of inner cities to the different needs of those living on the islands. The desire to offer as many services locally as is possible, weighed against the constant difficulty of maintaining skills from a small number of procedures, is a constant challenge. There is a perception, which may warrant further research, that a number of people with physical and mental health problems move to the islands for 'the good life', only to be presented with difficulties resulting from social isolation and remoteness from secondary care and other support services. If true, this could represent an additional workload for the island GP.

On the islands, single-handed practice needs by necessity, to be strengthened rather than victimised, which may be the case elsewhere as a result of the issues arising from Shipman.

In some ways island general practice maintains some of the best of 'old fashioned' primary care in terms of variety and continuity, while in other ways leading the way by the necessity to do as much as possible within primary care to avoid expensive and inconvenient trips off island.

The drawbacks are self-evident from the foregoing discussion and are largely the same 'advantageous' issues viewed from another aspect. Ultimately only individuals with the following characteristics need apply: self-motivated but able to network 'remotely', able to cultivate community relationships, flexible in taking on a variety of roles, prepared to 'have a go' where appropriate and necessary, able to think laterally about clinical and non-clinical issues, prepared to think outside usual boundaries, sound generalist training, and able to enjoy variety and continuity of care. In summary these jobs should suit any committed GP who wants to take their career in a new and stimulating direction.

\section{Jonathan R Clowes}

DOI: 10.3399/bjgp10X544186 